\title{
DIFFEOMORPHISMS OF THE TORUS WITH WANDERING DOMAINS
}

\author{
PATRICK D. MCSWIGGEN
}

(Communicated by Charles C. Pugh)

\begin{abstract}
Examples of $C^{3-\varepsilon}$ diffeomorphisms of the 2-torus are constructed, each of which is semiconjugate to an ergodic translation but has a wandering domain with dense orbit. This is a generalization of a classical example on the circle due to Denjoy.
\end{abstract}

In 1932 Denjoy [3] proved that any $C^{2}$ diffeomorphism of the circle with no periodic points had only dense orbits. In the same paper he also constructed a $C^{1}$ diffeomorphism of the circle, semiconjugate to an irrational rotation (and hence without periodic points), which had a 'wandering interval' with dense orbit. That is, there is a closed subinterval of the circle with the property that its iterates are disjoint, but the union of all are dense in the circle. (The first such example was due to Bohl [2].) This showed a clear break in the possible behaviour of a system depending on its differentiability. Harrison $[4,5]$ showed that these 'breaks in differentiability' occur in every dimension $\geq 2(\neq 4)$ and at every level of differentiability.

It is unknown whether a dichotomy similar to Denjoy's exists for diffeomorphisms of the 2-torus (or higher-dimensional tori), and if so, at what level of differentiability this break occurs. That is, does there exist $s>0$ such that for any $r<s$ but not for any $r \geq s$ there are $C^{r}$ diffeomorphisms of the torus with no periodic points which have a 'wandering disk' with dense orbit? Akis constructed a $C^{1}$ diffeomorphism of the torus with a densely wandering disk [1]. In contrast, Norton and Velling [11] showed that for a certain class of $C^{1}$ diffeomorphisms a wandering domain cannot exist.

In this paper we construct Denjoy-type diffeomorphisms of the 2-torus and conjecture that these represent an upper bound for the differentiability of such examples. Throughout we will follow the common practice whereby for any real number $r \geq 0$ a function is said to be of class $C^{r}$ if it possesses continuous derivatives of orders $\leq[r]$ and its $[r]$ th order derivative is $(r)$-Hölder. We prove the following.

Received by the editors March 27, 1991.

1991 Mathematics Subject Classification. Primary 54H20, 58E13; Secondary 58F15, 58F18.

Key words and phrases. Toral diffeomorphism, Denjoy counterexamples, wandering domain.

Research was conducted while the author was a Charles Phelps Taft Postdoctoral Fellow at the University of Cincinnati. 
Theorem. For every $\varepsilon>0$, there exists a $C^{3-\varepsilon}$ diffeomorphism of the 2-torus which has no periodic points, is semiconjugate to a translation, and has a wandering domain with dense orbit. That is, there exists an open set $O \subset \mathbb{T}^{2}$ such that $h^{i}(\bar{O}) \cap h^{j}(\bar{O})=\varnothing$ for all $i, j \in \mathbb{Z}$ with $i \neq j$, and $\bigcup_{i \in \mathbb{Z}} h^{i}(O)$ is dense in $\mathbb{T}^{2}$.

The first example of a general wandering domain (disjoint but recurrent iterates) for an arbitrary surface under a $C^{2+\delta}$ diffeomorphism was due to Harrison $[6,7]$. Here $\delta$ is bounded away from one. Harrison conjectured that given sufficient control over the distortion of higher iterates, $C^{3}$ may in general be an upper bound for this behavior.

The construction presented here occurs on the 3-torus. The basic procedure consists of a DA type construction on a $3 \times 3$ linear Anosov with a 1 dimensional unstable direction. The resulting system is shown to have a unique 1-dimensional $C^{3-\varepsilon}$ strong unstable foliation. The desired example is obtained as the holonomy map of this foliation on a 2-torus cross section. The initial step differs from a higher-dimensional DA in that it is the unstable rather than the stable direction which has dimension 1 . As a result, the perturbed system will not satisfy Axiom A; however, this is of no consequence since the perturbed system is promptly discarded in favor of only its strong unstable foliation anyway.

The construction has three parts. The first is to show there exist $3 \times 3$ linear Anosovs, that is, hyperbolic elements of $\mathrm{GL}_{3}(\mathbb{Z})$, with a single eigenvalue outside the unit circle and the remaining inside and equal in modulus. A $C^{\infty}$ modification of this is made which turns the saddle point into a repeller and which leaves the entire 3-torus as a pseudo-hyperbolic set with a 1-dimensional strong unstable direction everywhere close to the original unstable direction. This is sufficient to conclude the existence of a unique 1-dimensional $C^{\infty}$ strong unstable lamination of $\mathbb{T}^{3}$ which is nearly parallel to the original linear unstable foliation, and, in particular, is transverse to a 2-torus cross section. The holonomy map of this lamination from the cross section to itself defines a Denjoy homeomorphism of the torus. Finally, we observe that the construction can be performed to guarantee that this lamination is a $C^{3-\varepsilon}$ foliation, and therefore the holonomy map on the cross section is a $C^{3-\varepsilon}$ diffeomorphism.

\section{Construction of $h$}

To show that there exist elements in $\mathrm{GL}_{3}(\mathbb{Z})$ with the desired conditions on the eigenvalues, it is sufficient to show there are elements whose characteristic polynomial (degree 3 ) has a nonreal root off the unit circle. In that case, this root's conjugate pair must have the same modulus. Further, since the product of all three moduli is 1 , this pair must sit strictly on the opposite side of the unit circle from the remaining (necessarily real) eigenvalue. Therefore, either this matrix or its inverse has the desired properties.

A cubic polynomial will have exactly one real root if it has two critical values with strictly the same sign. Consider the family of (nonsymmetric) integer matrices:

$$
T_{a}=\left[\begin{array}{ccc}
0 & -1 & 0 \\
1 & a^{2}-1 & a \\
0 & a & 1
\end{array}\right], \quad a \in \mathbb{Z}-\{0\}
$$


The characteristic polynomial of $T_{a}$ is $p_{a}(x)=1+a^{2} x^{2}-x^{3}$. This polynomial has critical points at $x=0,2 a^{2} / 3$. The corresponding critical values are 1 , $1+4 a^{6} / 27$. Since both critical values are positive, $p_{a}(x)$ must have exactly one real root, $\lambda_{r}$. For $a \neq 0$, one can verify that $\lambda_{r}$ satisfies $1 \leq a^{2}<\lambda_{r}<a^{2}+a^{-4}$, and so the matrix is hyperbolic. We also observe that with $\operatorname{det}\left(T_{a}\right)=1$, these examples are orientation preserving.

Now suppose $T$ is any such matrix, and for convenience assume $T$ is orientation preserving. Let $g: \mathbb{T}^{3} \rightarrow \mathbb{T}^{3}$ be the induced Anosov diffeomorphism. Choose a coordinate chart $(\varphi, U)$ about the fixed point $p_{0} \in \mathbb{T}^{3}$ of $g$ such that $\varphi\left(p_{0}\right)=0$ and in local coordinates $g$ has the form $G \stackrel{\text { def }}{=}$ $\varphi \circ g \circ \varphi^{-1}: \varphi\left(U \cap g^{-1} U\right) \subset \mathbb{R} \times \mathbb{R}^{2} \rightarrow \mathbb{R} \times \mathbb{R}^{2}$ with $G(x, y)=\left(\lambda_{u} x, \lambda_{s} R y\right)$. Here $\lambda_{u}$ is the unstable eigenvalue of $T, \lambda_{s}$ is the modulus of the stable eigenvalues, and $R \in O_{2}(\mathbb{R})$ is a rotation in $\mathbb{R}^{2}$. Since $T$ is (always) nonsymmetric, its eigenspaces are not necessarily orthogonal and we cannot choose $T \varphi$ to be an isometry. Nevertheless, we can assume $T \varphi^{-1}$ preserves the relative lengths of vectors $(u, 0)$ and $(0, v)$. That is, $T \varphi^{-1}$ is an isometry on each factor of $\mathbb{R} \times \mathbb{R}^{2}$ separately up to a uniform scaling. By allowing uniform rescaling we can assume $B \stackrel{\text { def }}{=} I \times D \subset \varphi\left(U \cap g^{-1} U\right)$, where $I=[-1,1]$ and $D$ is the unit disk in $\mathbb{R}^{2}$.

We want to define a perturbation $F$ of $G$ such that $F \equiv G$ near $\partial B$, and 0 is a repelling fixed point of $F$. Anticipating future requirements, we will do this with the use of the following lemmas.

Lemma 1. Given $\alpha>\beta>0$, for any arbitrarily small $\kappa>0$, there exists $\lambda \in C^{\infty}(\mathbb{R}, \mathbb{R})$ such that $\lambda(t) \equiv \alpha$ for $t \leq 0, \lambda(t) \equiv \beta$ for $t \geq 1$, and $-\kappa / t \leq$ $\lambda^{\prime}(t)<0$ for $0<t<1$.

Proof. Let $t_{0}=\frac{1}{2} \exp [(\beta-\alpha) / \kappa]$. Since $(\beta-\alpha) / \kappa<0$, then $0<t_{0}<\frac{1}{2} . t_{0}$ is chosen such that the area under the graph of $y=\kappa / t$ on $\left[t_{0}, \frac{1}{2}\right]$ is $(\alpha-\beta)$. Let $\psi: \mathbb{R} \rightarrow \mathbb{R}$ be a $C^{\infty}$ function with a graph as in Figure 1: $\psi(t) \equiv 0$ for $t \leq 0$ or $t \geq 1, \psi(t) \equiv \kappa / t$ on $\left[t_{0}, \frac{1}{2}\right]$, and $0<\psi(t)<\kappa / t$ otherwise. By design, $\int_{[0,1]} \psi(s) d s>(\alpha-\beta)$. Hence, there exists $\tau<1$ such that the graph of $\tau \psi$ encloses exactly $(\alpha-\beta)$. Now define $\lambda$ by

$$
\lambda(t)=\alpha-\int_{0}^{t} \tau \psi(s) d s .
$$

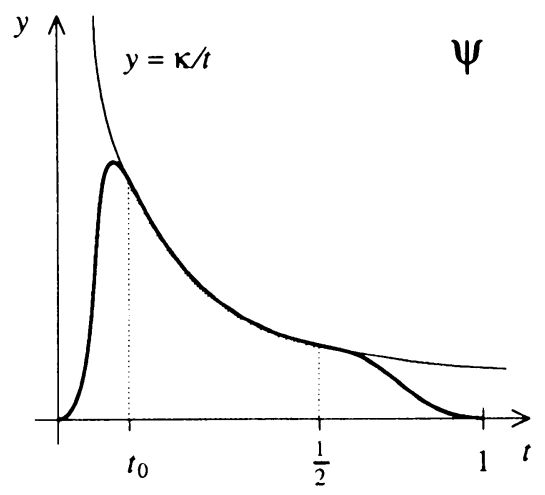

Figure 1 


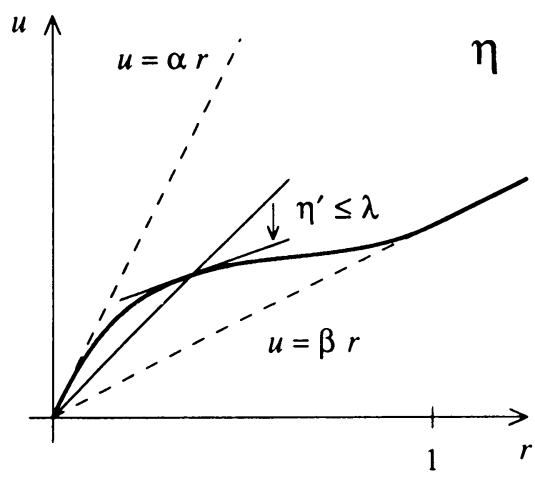

FIGURE 2

Given $\lambda$ as above, define a function $\eta: \mathbb{R} \rightarrow \mathbb{R}$ by $\eta(r) \stackrel{\text { def }}{=} r \lambda(r)$. We then have that $\eta(r) \equiv \alpha r$ for $r \leq 0$ and $\eta(r) \equiv \beta r$ for $r \geq 1$. See Figure 2. $\lambda(r)$ is the slope of the secant line connecting the origin to the point $(r, \eta(r))$ on the graph of $\eta$. Since $\lambda$ is decreasing on $[0,1]$, this implies that the graph of $\eta$ crosses lines through the origin from left to right; that is, $\eta^{\prime} \leq \lambda$. In fact, $\lambda-\eta^{\prime}=\lambda-\left(\lambda+r \lambda^{\prime}\right)=-r \lambda^{\prime}$, so $0 \leq\left(\lambda-\eta^{\prime}\right)(r) \leq \kappa$ for all $r$.

Since $\lambda$ is decreasing, the graph of $\eta$ sits above the line $u=\beta r$ for $r \geq 0$. The maximum deviation of $\eta$ from $u=\beta r \quad(r \geq 0)$ is $\sup _{r \geq 0}[\eta(r)-\beta r]$. Since $\lambda(1)=\beta$, it follows that $\eta(r)=r\left[\beta-\int_{1}^{r} \lambda^{\prime}(s) d s\right]$. Therefore, $\eta(r)-$ $\beta r=-r \int_{1}^{r} \lambda^{\prime}(s) d s \leq r \int_{1}^{r} \kappa / s d s=\kappa r \ln r$. On [0,1], $r \ln r \leq e^{-1}<1$. Consequently, $0 \leq \eta(r)-\beta r \leq \kappa$ for $r \geq 0$. This with the previous fact says that given $\alpha>\beta$, we can construct a smooth function $\eta$ such that $\eta$ is $C^{\infty}$ tangent to the line $u=\alpha r$ at the origin; within a fixed distance of 1 the graph of $\eta$ turns from this direction to be $C^{\infty}$ tangent to the line $u=\beta r$. This can be done so that not only is the deviation from the line $u=\beta r$ arbitrarily small, but the angle the graph makes with any ray through the origin (i.e., the rate of turning) is also arbitrarily small. We collect these as a lemma.

Lemma 2. Let $\lambda$ be a function satisfying the conclusions of Lemma 1, and let $\eta(r) \stackrel{\text { def }}{=} r \lambda(r)$. Then $0 \leq\left(\lambda-\eta^{\prime}\right)(r) \leq \kappa$ and $0 \leq \eta(r)-\beta r \leq \kappa$ for $r \geq 0$.

We can now construct our perturbation of $G$. Fix $\alpha$ with $1<\alpha<\lambda_{u}$, set $\beta=\lambda_{s}$, and let $\kappa>0$ be sufficiently small (to be specified as we proceed). Let $\lambda: \mathbb{R} \rightarrow \mathbb{R}$ be as in Lemma 1 , and $\eta(r)=r \lambda(r)$. Let $\chi: \mathbb{R} \rightarrow[0,1]$ be a $C^{\infty}$ bump function with $\chi(0)=1$ and $\chi(t) \equiv 0$ for $|t| \geq 1$. For simplicity, assume $\chi$ is even and $\chi^{\prime}(t)<0$ for $0<t<1$. Define $F: B \rightarrow \mathbb{R} \times \mathbb{R}^{2}$ by

$$
F(x, y)=\left(\lambda_{u} x, \lambda_{s} R y+\chi(x)\left[\lambda(\|y\|)-\lambda_{s}\right] R y\right) .
$$

Since $\lambda(\|y\|) \equiv \lambda_{s}$ for $\|y\| \geq 1$ and $\chi(x) \equiv 0$ for $|x| \geq 1$, it follows that $F \equiv G$ near $\partial B$. Moreover, the assumption $\lambda(r) \equiv \alpha$ for $r \leq 0$ guarantees that $\lambda(\|y\|)$ is $C^{\infty}$, which implies $F$ is $C^{\infty}$.

We claim that if $\kappa$ is sufficiently small, then $F$ remains an embedding. In adapted cylindrical coordinates $F$ has the form

$$
(x, r, \theta) \mapsto\left(\lambda_{u} x, \lambda_{s} r+\chi(x)\left[\eta(r)-\lambda_{s} r\right], \theta+\theta_{0}\right) .
$$




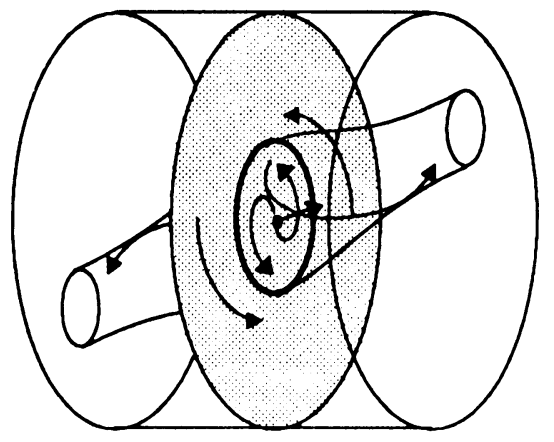

FIGURE 3

Clearly $F$ is invertible $\Leftrightarrow$ for each $x, r \mapsto \lambda_{s} r+\chi(x)\left[\eta(r)-\lambda_{s} r\right]$ is invertible. This has derivative $(1-\chi) \lambda_{s}+\chi \eta^{\prime}$. From the previous lemmas, $\eta^{\prime} \geq \lambda(r)-\kappa \geq$ $\lambda_{s}-\kappa$. Hence, $(1-\chi) \lambda_{s}+\chi \eta^{\prime} \geq \lambda_{s}-\kappa$, which is positive for small $\kappa$. Consequently, $F$ is invertible, and combining this with $F \equiv G$ on $\partial B$, we have $\operatorname{image}(F)=\operatorname{image}(G)$. Therefore, we can define a smooth diffeomorphism $f: \mathbb{T}^{3} \rightarrow \mathbb{T}^{3}$ by $f=\varphi^{-1} \circ F \circ \varphi$ on $\varphi^{-1}(B)$ and $f=g$ on $\varphi^{-1}(B)^{c}$.

This perturbation turns the saddle point of $g$ into a repelling fixed point surrounded by a normally hyperbolic circle $S$ located (in $\varphi$ coordinates) at $x=0$, and $\|y\|=c$, where $c$ is the fixed point of $\eta(\lambda(c)=1)$. The unstable manifold of $S$ forms the boundary of the basin of repulsion of the fixed point $p_{0}$ (Figure 3 ). In character, this system looks like the end result of a Hopf bifurcation. In fact, if $\alpha$ is treated as a variable parameter ranging from $\alpha=\lambda_{s}$ to $\alpha>1$, then this can be constructed to give a 1-parameter family of diffeomorphisms connecting $g$ to $f$ with a Hopf bifurcation at $\alpha=1$.

We want to show $\mathbb{T}^{3}$ is a pseudo-hyperbolic set for $f$ provided $\kappa$ is sufficiently small. Let $T \mathbb{T}^{3}=E_{1} \oplus E_{2}$ be the original hyperbolic splitting for $g$. For $p \notin \varphi^{-1}(B), T_{p} f=T_{p} g$, so $E_{1} \oplus E_{2}$ is invariant with $T_{p} f$ expanding vectors in $E_{1 p}$ by $\lambda_{u}$ and contracting vectors in $E_{2 p}$ by $\lambda_{s}$. If $p \in \varphi^{-1}(B)$, we calculate the local coordinates. The derivative of $F=\left.\varphi \circ f \circ \varphi^{-1}\right|_{\varphi^{-1} B}$ is

$$
(D F)_{(x, y)}=\left[\begin{array}{cc}
\lambda_{u} & 0 \\
\varepsilon(x, y) & N_{(x, y)}
\end{array}\right]
$$

where $N_{(x, y)} \in L\left(\mathbb{R}^{2}\right)$ is defined by

$$
N_{(x, y)} v=\left[\lambda_{s}+\left(\lambda-\lambda_{s}\right) \chi\right] R v+\chi \lambda^{\prime}\|y\|^{-1}(y \cdot v) R y
$$

and $\varepsilon(x, y)=\left(\lambda-\lambda_{s}\right) \chi^{\prime} R y$. Here $\chi, \chi^{\prime}$ are evaluated at $x$, and $\lambda, \lambda^{\prime}$ at $\|y\|$. If $v \perp y$, then $N_{(x, y)} v=\left[(1-\chi) \lambda_{s}+\chi \lambda\right] R v$. On the other hand, if $v \| y$, then $(y \cdot v) R y=(y \cdot y) R v$. Hence, $N_{(x, y)} v=\left[(1-\chi) \lambda_{s}+\chi\left(\lambda+\|y\| \lambda^{\prime}\right)\right] R v$. Since $\lambda(\|y\|)+\|y\| \lambda^{\prime}(\|y\|)=\eta^{\prime}(\|y\|)$, it follows that $v \| y$ implies $N_{(x, y)} v=$ $\left[(1-\chi) \lambda_{s}+\chi \eta^{\prime}\right] R v$.

Any vector can be decomposed into parts parallel and perpendicular to $y$. Therefore, since $\|R\|=1$ and $\eta^{\prime} \leq \lambda$, we have for any $v \in \mathbb{R}^{2}$,

$$
\left[(1-\chi) \lambda_{s}+\chi \eta^{\prime}\right]\|v\| \leq\left\|N_{(x, y)} v\right\| \leq\left[(1-\chi) \lambda_{s}+\chi \lambda\right]\|v\| .
$$

Also, $\|\varepsilon(x, y)\|=\left[\|y\| \lambda(\|y\|)-\lambda_{s}\|y\|\right]\left|\chi^{\prime}(x)\right|=\left[\eta(\|y\|)-\lambda_{s}\|y\|\right]\left|\chi^{\prime}(x)\right| \leq \kappa\|\chi\|_{C^{1}}$. Hence, by choosing $\kappa$ sufficiently small, we can assume $(D F)_{(x, y)}$ is as close 
as we like to diagonal. Since $T_{p} \varphi: E_{1 p} \oplus E_{2 p} \rightarrow \mathbb{R} \times \mathbb{R}^{2}$ is an isometry on each factor (up to uniform scaling), we can conclude $T_{p} f$ has the same form as $(D F)_{(x, y)}$ with these same estimates.

Define $\lambda_{1}, \lambda_{2}: \mathbb{T}^{3} \rightarrow \mathbb{R}$ as follows. $\lambda_{1}(p)=\lambda_{2}(p)=\lambda_{s}$ for $p \notin \varphi^{-1}(B)$. For $p \in \varphi^{-1}(B)$ with $(x, y)=\varphi(p), \lambda_{1}(p)=\left[(1-\chi) \lambda_{s}+\chi \eta^{\prime}\right]$ and $\lambda_{2}(p)=$ $\left[(1-\chi) \lambda_{s}+\chi \lambda\right]$. Here again $\chi$ is evaluated at $x$, and $\lambda, \eta^{\prime}$ at $\|y\|$. Since $\lambda, \eta^{\prime} \equiv \lambda_{s}$ for $\|y\| \geq 1$, we have $\lambda_{1}, \lambda_{2}$ are $C^{\infty}$. Further, $\lambda_{1}(p) \leq \lambda_{2}(p)$ for all $p$, support $\left(\lambda_{2}-\lambda_{1}\right) \subset \varphi^{-1}(B)$, and $\left(\lambda_{2}-\lambda_{1}\right)(p) \leq \kappa$. Since $\eta^{\prime} \geq \lambda_{s}-\kappa$ and $\alpha \geq \lambda$, it follows that $0<\lambda_{s}-\kappa \leq \lambda_{1}(p) \leq \lambda_{2}(p) \leq \alpha$.

Combining the results for $p \in \varphi^{-1}(B)$ and $p \notin \varphi^{-1}(B)$, we have that relative to the splitting $E_{1} \oplus E_{2}, T_{p} f$ has the form

$$
T_{p} f=\left[\begin{array}{cc}
A_{p} & 0 \\
C_{p} & N_{p}
\end{array}\right]
$$

where $\left\|A_{p} u\right\|=\lambda_{u}\|u\|,\left\|C_{p}\right\| \leq \kappa\|\chi\|_{C^{1}}$, and $\lambda_{1}(p)\|v\| \leq\left\|N_{p} v\right\| \leq \lambda_{2}(p)\|v\|$.

$T_{p} f$ leaves the plane bundle $E_{2}$ invariant. Although $T_{p} f$ is not a contraction on $E_{2}$, it expands vectors by no more than $\alpha<\lambda_{u}$. Set $E^{p s}=E_{2}$. $E_{1}$ is no longer invariant, but we claim there is a new invariant line bundle $E^{u}$ close to $E_{1}$ which $T_{p} f$ expands by not much less than $\lambda_{u}$. Any line in $T_{p} M$ which is close to $E_{1 p}$ is the graph of a unique element of $L\left(E_{1 p}, E_{2 p}\right)$. Let $\pi: \mathscr{L}\left(E_{1}, E_{2}\right) \rightarrow \mathbb{T}^{3}$ be the standard vector bundle over $\mathbb{T}^{3}$ with fibers $\pi^{-1}(p)=L\left(E_{1 p}, E_{2 p}\right)$. A continuous section of $\mathscr{L}\left(E_{1}, E_{2}\right)$ corresponds to a continuous line field on $\mathbb{T}^{3}$. We want to show there is a $T f$ invariant section in $\mathscr{L}\left(E_{1}, E_{2}\right)$.

The induced action of $T f$ on $\mathscr{L}\left(E_{1}, E_{2}\right)$ is as follows. Given $S_{p} \in$ $L\left(E_{1 p}, E_{2 p}\right),\left[I_{1 p}, S_{p}\right]: E_{1 p} \rightarrow T_{p} \mathbb{T}^{3}$ has as its image the graph of $S_{p}=\operatorname{gr}\left(S_{p}\right)$. Therefore, $T_{p} f\left(\operatorname{gr}\left(S_{p}\right)\right)$ is the image of

$$
T_{p} f \circ\left[I_{1 p}, S_{p}\right]=\left[A_{p}, C_{p}+N_{p} S_{p}\right]=\left[I_{1 f p}, C_{p} A_{p}^{-1}+N_{p} S_{p} A_{p}^{-1}\right] \circ A_{p} .
$$

Consequently, $\left[I_{1 f p}, C_{p} A_{p}^{-1}+N_{p} S_{p} A_{p}^{-1}\right]: E_{1 f p} \rightarrow T_{f p} \mathbb{T}^{3}$ has the same image $\Rightarrow$ $S_{p} \mapsto C_{p} A_{p}^{-1}+N_{p} S_{p} A_{p}^{-1}$ is the induced action of $T f$ on the fiber $L\left(E_{1 p}, E_{2 p}\right)$. Therefore, $T f$ contracts $L\left(E_{1 p}, E_{2 p}\right)$ by $\left\|N_{p}\right\| \cdot\left\|A_{p}^{-1}\right\| \leq \alpha / \lambda_{u}$. The space of bounded sections of $\mathscr{L}\left(E_{1}, E_{2}\right)$ with the sup norm is a Banach space which $T f$ contracts by $\alpha / \lambda_{u}<1$. Consequently, there is a unique continuous $T f$ invariant section $\sigma_{u} \in \mathscr{L}\left(E_{1}, E_{2}\right)$. Let $E^{u}$ be the corresponding continuous $T f$ invariant line field; then $E^{u} \oplus E^{p s}$ is a continuous $T f$ invariant splitting of $T \mathbb{T}^{3}$.

Since the image of the zero section is bounded in norm by $\sup \left\|C_{p}\right\| \cdot\left\|A_{p}^{-1}\right\| \leq$ $\kappa\|\chi\|_{C^{1}} \lambda_{u}^{-1}, T f$ takes the ball of radius $k=\kappa\|\chi\|_{C^{\prime}}\left(\lambda_{u}-\alpha\right)^{-1}$ into itself. Therefore, $\left\|\sigma_{u}\right\| \leq k$, which implies that for each $p, E_{p}^{u}$ is contained in the cone $\left\{(u, v) \in T_{p} \mathbb{T}^{3}:\|v\| \leq k\|u\|\right\}$. Choose $\kappa$ so that $k \leq 1$, and define an equivalent Finsler on $T \mathbb{T}^{3}$ by $\|(u, v)\|=\max \{\|u\|,\|v\|\}$ for $(u, v) \in E_{1} \oplus E_{2}$. Under this Finsler $\|(u, v)\|=\|u\|$ for $(u, v) \in E^{u}$. Since $E^{u}$ is invariant $\Rightarrow T_{p} f(u, v) \in E^{u}$ and so $\left\|T_{p} f(u, v)\right\|=\left\|A_{p} u\right\|=\lambda_{u}\|(u, v)\|$. Therefore, $T_{p} f$ expands $E^{u}$ by $\lambda_{u}$ and consequently, for $\rho$ with $\alpha<\rho<\lambda_{u}$, all of $\mathbb{T}^{3}$ is a $\rho$-pseudo-hyperbolic set for $f$ with strong unstable direction $E^{u}$ (since $\rho>\alpha>1$ ). 
The line field $E^{u}$ will integrate to a $C^{r} f$-invariant foliation $\mathscr{F}$ if $E^{u}$ is $C^{r}$. We claim that $f$ can be constructed so that $E^{u}$ will be $C^{3-\varepsilon}$ for any $\varepsilon>0$. For this we will use the $C^{r}$ Section Theorem, which we state here in abbreviated form.

Theorem 3 ( $C^{r}$ Section Theorem [9]). Let $\pi: L \rightarrow X$ be a finite-dimensional Finslered vector bundle over a compact set. Let $F: L \rightarrow L$ be continuous, fiber preserving, and cover a homeomorphism $f: X \rightarrow X$. Suppose, for all $x \in X$, the restriction of $F$ to the fiber over $x, F_{x}: L_{x} \rightarrow L_{f x}$, is Lipschitz and $l_{x} \stackrel{\text { def }}{=}$ $\operatorname{Lip}\left(F_{x}\right)<1$. Then there exists a unique bounded section $\sigma: X \rightarrow L$ such that $\sigma$ is continuous and $F \circ \sigma=\sigma \circ f$ (an invariant section). If $L$ and $X$ are $C^{r}$ manifolds $(r \geq 1), F$ is $C^{r}, f$ is a $C^{r}$ diffeomorphism, and for some Finsler on $T X, \tau_{x} \stackrel{\text { def }}{=}\left\|\left(T_{x} f\right)^{-1}\right\|$ satisfies $l_{x}\left(\tau_{x}\right)^{r}<1$ for each $x$, then $\sigma$ is $C^{r}$.

Fix $\varepsilon>0$. We have already observed that $l_{p}=\left\|N_{p}\right\| \cdot\left\|A_{p}^{-1}\right\|=\lambda_{2}(p) / \lambda_{u}<1$. We must compare $l_{p}$ to $\tau_{p}=\left\|\left(T_{p} f\right)^{-1}\right\|$. Define an equivalent metric on $M$ by declaring $E_{p}^{u} \perp E_{p}^{p s}$. (Note: we do not change the Finsler on $\mathscr{L}\left(E_{1}, E_{2}\right)$ derived from the original metric, so $l_{p}$ is unchanged. Both are independent of the Finsler defined earlier to show that $\mathbb{T}^{3}$ is pseudo-hyperbolic.) Relative to this metric, $\left\|\left(T_{p} f\right)^{-1}\right\|=\left\|N_{p}^{-1}\right\|=\lambda_{1}(p)^{-1}$. Consequently, to guarantee that $E^{u}$ is $C^{3-\varepsilon}$, we need $f$ to be constructed so that $\left[\lambda_{2} \lambda_{u}^{-1} \lambda_{1}^{-3+\varepsilon}\right](p)<1$.

Observe that for the linear system, $\lambda_{1}(p)=\lambda_{2}(p) \equiv \lambda_{s}$ and $\lambda_{u}^{-1}=\lambda_{s}^{2}$. Hence, for $g,\left[\lambda_{2} \lambda_{u}^{-1} \lambda_{1}^{-3+\varepsilon}\right](p) \equiv \lambda_{s}^{\varepsilon}<1$. The object is then to retain this property when perturbing $g$. Note that for $\varepsilon=0$, even the linear system fails to satisfy this condition, and therefore it would be hopeless to try to use this method to construct a $C^{3}$ example.

Set $\delta=\varepsilon / 2$. We have $\lambda_{1}(p) \geq \lambda_{s}-\kappa$, so we can choose $\kappa$ sufficiently small that $\lambda_{1}(p) \geq \lambda_{s}^{1+\delta}$. We also have $\lambda_{u}=\lambda_{s}^{-2}$. Therefore,

$$
\begin{aligned}
\left(\frac{\lambda_{2}}{\lambda_{u}}\right)\left(\frac{1}{\lambda_{1}}\right)^{3-\varepsilon} & =\left(1+\frac{\lambda_{2}-\lambda_{1}}{\lambda_{1}}\right)\left(\frac{1}{\lambda_{u}}\right)\left(\frac{1}{\lambda_{1}}\right)^{2-\varepsilon} \\
& \leq\left(1+\frac{\kappa}{\lambda_{s}^{1+\delta}}\right) \lambda_{s}^{2}\left(\lambda_{s}^{1+\delta}\right)^{-2+\varepsilon} \leq\left(1+\frac{\kappa}{\lambda_{s}^{2}}\right) \lambda_{s}^{\varepsilon^{2} / 2} .
\end{aligned}
$$

By choosing $\kappa$ possibly smaller this can be made less than 1 since $\lambda_{s}^{\varepsilon^{2} / 2}<1$. Therefore, by the $C^{r}$ Section Theorem, $E^{u}$ is $C^{3-\varepsilon}$, and, consequently, $\mathscr{F}$ is a $C^{3-\varepsilon} f$-invariant foliation.

From general Stable Manifold Theory [9], the leaf of $\mathscr{F}$ through $p$ is the strong unstable manifold of $p$ for $f, W^{u}(p, f)$. This is a 1-to-1 $C^{\infty}$ immersed copy of $\mathbb{R}$. Therefore, $\mathscr{F}$ has no compact leaves. The original foliation of $\mathbb{T}^{3}$ by the unstable manifolds of $g$ has a global cross section $\Sigma \cong \mathbb{T}^{2}$ corresponding to the image under the covering map $\mathbb{R}^{3} \rightarrow \mathbb{T}^{3}$ of a coordinate plane $P_{0}$ transverse to the unstable eigenvector of $T$. If $k$ is sufficiently small (implying $\kappa$ is sufficiently small), the lift of $\mathscr{F}$ to $\mathbb{R}^{3}$ will be everywhere transverse to the family of planes parallel to $P_{0}$. Consequently, $\Sigma$ is a global cross section for $\mathscr{F}$ and the holonomy map $h: \Sigma \rightarrow \Sigma$ is a well-defined homeomorphism. $\left(\mathbb{T}^{3} \cong S^{1} \times \Sigma\right.$. Therefore, there are actually two possible choices for $h$, one being the inverse of the other.) Since $\mathscr{F}$ is a $C^{3-\varepsilon}$ foliation, $h$ is a $C^{3-\varepsilon}$ diffeomorphism. 


\section{VERIFICATION OF PROPERTIES}

We are left with showing that $h$ has the qualitative behavior that we want. $h$ clearly cannot have any periodic points since this would correspond to a compact leaf of $\mathscr{F}$. Let $p_{0}$ be the repelling fixed point of $f, V$ the basin of repulsion of $p_{0}$, and $O$ any connected component of $V \cap \Sigma$. The orbit of $O$ under $h$ is exactly the collection of all connected components of $V \cap \Sigma$. Further, $V$ is bounded by the unstable manifold of the circle $S$. This unstable manifold also cannot intersect itself. Hence, the iterates of $\bar{O}$ are disjoint.

We claim that the orbit of $O$ is dense. Let $q_{0} \in \Sigma$ be any point and $W_{0} \subset \Sigma$ any neighborhood of $q_{0}$. Choose a foliation box $W$ for $\mathscr{F}$ with $W \cap \Sigma=W_{0}$. Let $L_{0}$ be the stable manifold of $g$ passing through $q_{0}$ and $\Delta \subset L_{0} \cap W$ a disk about $q_{0}$. Since $W$ is a foliation box and $V$ is the union of leaves of $\mathscr{F}$, if $W \cap V \supset \Delta \cap V \neq \varnothing$, then $V$ intersects $W_{0}$. Hence, $h^{n}(O) \cap W_{0} \neq \varnothing$ for some $n$ and the orbit of $O$ is dense.

Since $V$ is $f$-invariant, to show $\Delta \cap V \neq \varnothing$ it is sufficient to show $f^{-n}(\Delta) \cap$ $V \neq \varnothing$ for some $n$. We first observe that there exists $r>0$ such that a subdisk $D$ of radius $r$ of any stable manifold of $g$ must intersect the strong unstable manifold of $p_{0}$ for $f$. Since this is contained in $V$, any such disk must also intersect $V$. To see this, lift $f$ to $\mathbb{R}^{3}$. A disk in a stable manifold of $g$ corresponds to a (flat) 2-disk in $\mathbb{R}^{3}$ centered on a point in the unit cube and parallel to the stable eigenspace of $T$. The lift of the strong unstable manifold of $p_{0}$ for $f$ is contained in a narrow cone (depending on $k$ ) of the unstable eigenspace of $T$. Further, this cone was constructed relative to the stable/unstable splitting, and so there is a lower bound $\theta>0$ to the angle that any line contained in this coise makes with the stable eigenspace. Now let $w$ be any point in the unit cube, $P$ the plane through $w$ parallel to the stable eigenspace, and $l$ any line contained in this cone. Since there is a lower bound on the angle that $l$ makes with $P$ and an upper bound on the distance from $w$ to $l$ ( $l$ contains points in the unit cube), there is also an upper bound $r$ on the distance from $w$ to the point of intersection $l \cap P$. Therefore, any such disk of radius $r$ must cut completely across this cone and so must intersect the strong unstable manifold of $p_{0}$ for $f$.

Since the pertubation of $g$ to get $f$ was along $g$-stable manifolds, $f$ leaves these invariant. Consequently, if $\Delta$ is contained in a $g$-stable manifold, then the same holds for all $f^{-n}(\Delta)$. If $f^{-1}$ expands disks in the $g$-stable direction, then for $n$ sufficiently large $f^{-n}(\Delta)$ will contain a disk of radius $r$ and, hence, must intersect $V$. Let $K=\left\{p \in \mathbb{T}^{3}: \lambda_{2}(p) \geq 1\right\}$. This is a closed neighborhood of $p_{0}$ containing the invariant circle $S$ (where $\lambda_{2} \equiv 1$ ). Since $\lambda_{1} \leq \lambda_{2}<1$ on $K^{c}, f^{-1}$ expands in the $g$-stable direction on $K^{c}$ (albeit nonuniformly). We want to show $K-S \subset V$, and therefore $f^{-1}$ will expand disks in the $g$-stable direction off a set contained in $V$. Since $\lambda_{2} \equiv \lambda_{s}<1$ on $\varphi^{-1}(B)^{c}, K \subset \varphi^{-1}(B)$. Returning to local coordinates, one has $\varphi(K)$ defined by $\left(\lambda_{2} \circ \varphi^{-1}\right)(x, y)=\lambda_{s}+\chi(x)\left[\lambda(\|y\|)-\lambda_{s}\right] \geq 1$. Since the only $y$ dependence comes as $\|y\|, \varphi(K)$ is symmetric with respect to rotation about the $x$-axis. (With $\chi$ even, it is also symmetric with respect to reflection in the $y$-plane.)

By construction, $\chi$ and $\lambda$ are strictly decreasing functions of $|x|$ and $\|y\|$ respectively on $0 \leq|x|$ and $\|y\| \leq 1$. Further, $\lambda \geq \lambda_{s}$. Therefore, $\lambda_{s}+\chi(x)\left[\lambda(\|y\|)-\lambda_{s}\right]$ strictly increases as $|x|$ and $\|y\|$ decrease. This implies 


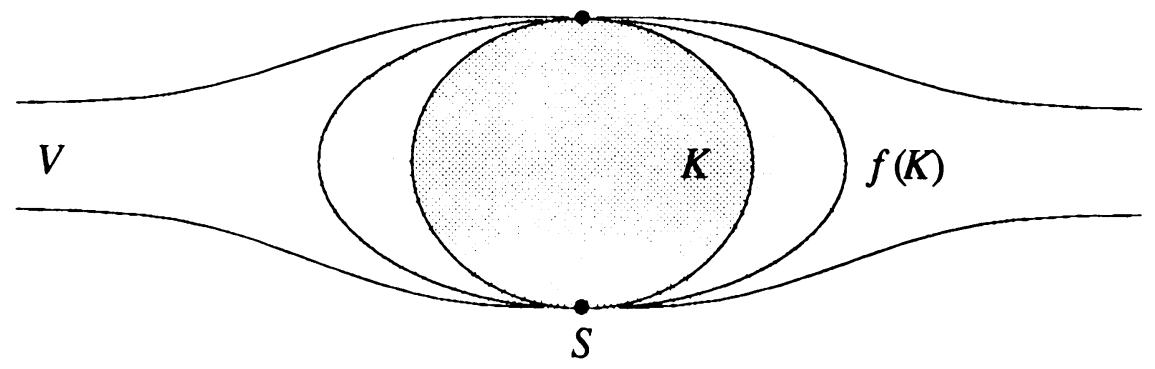

FIGURE 4

that, first, $\partial \varphi(K)$ is precisely the level set $\lambda_{2} \circ \varphi^{-1}=1$, and, second, $\partial \varphi(K)$ is a surface of revolution (from symmetry) generated by a strictly decreasing function. Equivalently, if $C(t, r)$ denotes the solid cylinder $\{(x, y):|x| \leq t,\|y\| \leq$ $r\}$, then $\varphi(K) \supset C\left(\left|x_{0}\right|,\left\|y_{0}\right\|\right)$ for any $\left(x_{0}, y_{0}\right) \in \varphi(K)$, and $C\left(\left|x_{0}\right|,\left\|y_{0}\right\|\right)$ is contained in $\operatorname{int}[\varphi(K)]$ unless $\left(x_{0}, y_{0}\right) \in \partial \varphi(K)$ when it misses the two circles $\left\{(x, y): x= \pm x_{0},\|y\|=\left\|y_{0}\right\|\right\}$.

Since $F(x, y)=\left[\lambda_{u} x,\left(\lambda_{2} \circ \varphi^{-1}\right)(x, y) R y\right]$ and $\left(\lambda_{2} \circ \varphi^{-1}\right)(x, y)=1$ on $\partial \varphi(K)$, then $F(x, y)=\left[\lambda_{u} x, R y\right]$ on $\partial \varphi(K) . R$ is a rotation about the $x$-axis and $\partial \varphi(K)$ is symmetric with respect to rotation about the $x$-axis. Therefore, $F$ just scales $\partial \varphi(K)$ by $\lambda_{u}$ in the $x$ direction. Since $\lambda_{u}>1$, all points in $\partial \varphi(K)$ are moved strictly away from the plane $x=0$ except for $\{x=$ $0\} \cap \partial \varphi(K)=\varphi(S)$. Therefore, it follows that int $[F \circ \varphi(K)] \supset \varphi(K)-\varphi(S)$, and conversely $F^{-1}[\varphi(K)-\varphi(S)] \subset \operatorname{int}[\varphi(K)] \Rightarrow f^{-1}(K-S) \subset \operatorname{int}[K]$.

Suppose $\left(x_{0}, y_{0}\right) \in \operatorname{int}[\varphi(K)]$. Then $\left\{(x, y):|x| \leq\left|x_{0}\right|,\|y\| \leq\left\|y_{0}\right\|\right\} \subset$ $\operatorname{int}[\varphi(K)]$. Since this does not intersect $\partial \varphi(K), \lambda_{2} \circ \varphi^{-1}$ is bounded strictly below 1 on this set. Therefore, $F^{-1}$ uniformly contracts this cylinder to the origin, which implies $\left(x_{0}, y_{0}\right) \in \varphi(V)$, and hence, $\operatorname{int}(K) \subset V$. But then $f^{-1}(K-S) \subset \operatorname{int}[K] \subset V \Rightarrow K-S \subset f(V)=V$ (see Figure 4).

Now suppose the radius of $\Delta$ is $d$. $f$ preserves the stable foliation of $g$ and induces the same map on the space of leaves. Therefore, $f^{-1}(\Delta)$ is contained in a stable manifold of $g$ and contains a disk of radius $d^{\prime}$. We want to show $d^{\prime}>d$. We can assume $f^{-1}(\Delta)$ does not intersect int $[f(K)]$, for otherwise it already intersects $V$ and we are done. Define $O$ to be the union of $\operatorname{int}[f(K)]$ with the set of all points less than $\delta<d / 2$ away from $K$ along a stable manifold of $g$. The latter set is the union of relatively open disks but is not itself open because of the two "end points" of $K$ corresponding to $\|y\|=0$. However, for $\delta$ sufficiently small these are contained in $\operatorname{int}[f(K)]$, and so $O$ is an open neighborhood of $K$. Therefore, $O^{c}$ is a compact set not intersecting $K \Rightarrow v=\sup \left\{\lambda_{2}(p): p \notin O\right\}<1$.

Let $L^{\prime}$ be the stable manifold of $g$ containing $f^{-1}(\Delta)$, and let $d_{s}$ be the distance in $L^{\prime}$ between points of $L^{\prime}$. Define $d^{\prime}=d_{s}\left[\partial f^{-1}(\Delta), f^{-1} q_{0}\right]$ and let $\Delta^{\prime}$ be the disk in $L^{\prime}$ of radius $d^{\prime}$ about $f^{-1} q_{0}$. Then $\Delta^{\prime} \subset f^{-1}(\Delta)$. Choose $q_{1} \in \partial f^{-1}(\Delta)$ with $d_{s}\left(q_{1}, f^{-1} q_{0}\right)=d^{\prime}$. We have assumed $f^{-1}(\Delta)$ does not intersect int $[f(K)]$, so either $\Delta^{\prime}$ is contained in $O^{c}$, or $\Delta^{\prime}$ intersects $O-\operatorname{int}[f(K)]$. In the geometry of $L^{\prime}$, all intersections of $L^{\prime}$ with $O-\operatorname{int}[f(K)]$ are round annuli of width at most $\delta$. Since by assumption $\Delta^{\prime}$ does not overlap the inner disk of any such annulus, any radius of $\Delta^{\prime}$ can have at most the outer 
segment of length $\delta$ contained in $O-\operatorname{int}[f(K)]$, with the remaining $d^{\prime}-\delta$ contained in $O^{\mathfrak{c}}$. In particular, this holds for the radius connecting $q_{1}$ and $f^{-1}\left(q_{0}\right)$.

For $p \in O-\operatorname{int}[f(K)], \lambda_{2}(p) \leq 1$. For $p \in O^{c}, \lambda_{2}(p) \leq \nu$. Since $\left\|\left.T_{p} f\right|_{E_{2 p}}\right\|=\lambda_{2}(p)$, we can use the Mean Value Theorem to obtain

$$
d_{s}\left(q_{0}, f\left(q_{1}\right)\right) \leq \delta+\nu\left(d^{\prime}-\delta\right) .
$$

On the other hand, $f\left(q_{1}\right) \in \partial \Delta \Rightarrow d_{s}\left(q_{0}, f\left(q_{1}\right)\right)=d$. Therefore, we have $d^{\prime} \geq \nu^{-1}(d-\delta)+\delta$. Since $\delta<d / 2$, it follows that $d^{\prime}>\left[1+(2 \nu)^{-1}\right] d$ and $f^{-1}$ expands $\Delta$ by at least $\left[1+(2 \nu)^{-1}\right]>1$.

We can now repeat this to the disk $\Delta^{\prime}$. Since $\delta<d / 2<d^{\prime} / 2$, either $f^{-2}(\Delta)$ intersects $\operatorname{int}[f(K)] \subset V$, or $f^{-2}$ expands $\Delta$ by at least $\left[1+(2 \nu)^{-1}\right]^{2}$. Repeating, we have that either $f^{-n}(\Delta)$ contains a disk of radius $\left[1+(2 \nu)^{-1}\right]^{n} d$, or it intersects $V$. However, $\left[1+(2 \nu)^{-1}\right]^{n} d \geq r$ for $n$ sufficiently large, which also implies $f^{-n}(\Delta)$ intersects $V$. This is precisely what we needed to show that the orbit of $O$ is dense.

We are left with showing that $h$ is semiconjugate to a translation. We observe that since the unstable foliation of $g$ is the image of a family of parallel lines under the covering map, the holonomy map thus induced on $\Sigma$ is a translation. Denote this translation by $\tau$ (assume compatible orientations on the leaves for defining $h$ and $\tau$ ). We claim $\tau$ is semiconjugate to $h$. To show this we use the following result of Walters.

Theorem 4 [12]. Let $g$ be an Anosov diffeomorphism of a compact manifold $M$. For any $\varepsilon>0$, there exists $\delta>0$ such that if $f$ is a homeomorphism of $M$ which is $\delta C^{0}$-close to $g$, then $f$ is semiconjugate to $g, \zeta \circ f=g \circ \zeta, b y$ a continuous function $\zeta: M \rightarrow M$ which is $\varepsilon C^{0}$-close to the identity.

For the example in hand, the $C^{0}$ size of the perturbation is controlled by $\kappa$ since $\|(F-G)(x, y)\|=\left|\chi(x)\left[\eta(\|y\|)-\lambda_{s}\|y\|\right]\right| \leq \kappa\|\chi\|_{C^{0}}$ by Lemma 2 . Therefore, by choosing $\kappa$ sufficiently small, we can assume there is a semiconjugacy from $f$ to $g$ which is as $C^{0}$-close to the identity as we like.

Let $U$ be a small enough neighborhood of $\Sigma$ that the projection of $U$ onto $\Sigma$ along the unstable foliation of $g, \pi_{u}: U \rightarrow \Sigma$, is well defined. If the semiconjugacy $\zeta$ from $f$ to $g$ is sufficiently close to the identity, then $\zeta(\Sigma) \subset U$. Assume $\kappa$ is small enough that this holds, and define $\omega: \Sigma \rightarrow \Sigma$ by $\omega=\left.\pi_{u} \circ \zeta\right|_{\Sigma}$. The unstable foliation of $g$ is by parallel lines, so $\pi_{u}$ is smooth $\Rightarrow \omega$ is continuous. We want to show $\omega \circ h=\tau \circ \omega$.

We first observe that $\zeta$ takes strong unstable manifolds of $f$ injectively to unstable manifolds of $g$. Assume $p \neq q$ are points on the same $f$ unstable manifold. For some $n>0, d\left(f^{n}(p), f^{n}(q)\right)$ will be on the order of the diameter of $\mathbb{T}^{3}$. This implies $\zeta \circ f^{n}(p) \neq \zeta \circ f^{n}(q)$ since $\zeta$ is close to the identity. Since $\zeta \circ f=g \circ \zeta$, it follows that $\zeta \circ f^{n}=g^{n} \circ \zeta$ for all $n$. Therefore, $g^{n} \circ \zeta(p) \neq g^{n} \circ \zeta(q) \Rightarrow \zeta(p) \neq \zeta(q)$, and $\zeta$ is injective.

Suppose $\zeta$ takes $p$ and $q$ to different $g$ unstable manifolds. Since $\mathbb{T}^{3}$ is hyperbolic for $g, g^{-1}$ expands the distance between $\zeta(p)$ and $\zeta(q)$. For any $N>0$, there exists $n \geq N$ such that $g^{-n} \circ \zeta(p)$ and $g^{-n} \circ \zeta(q)$ will be on the order of the diameter of $\mathbb{T}^{3}$ apart. On the other hand, for $N$ sufficiently large, $d\left(f^{-n}(p), f^{-n}(q)\right)$ is as small as we like, which implies $\zeta \circ f^{-n}(p)$ and $\zeta \circ$ 


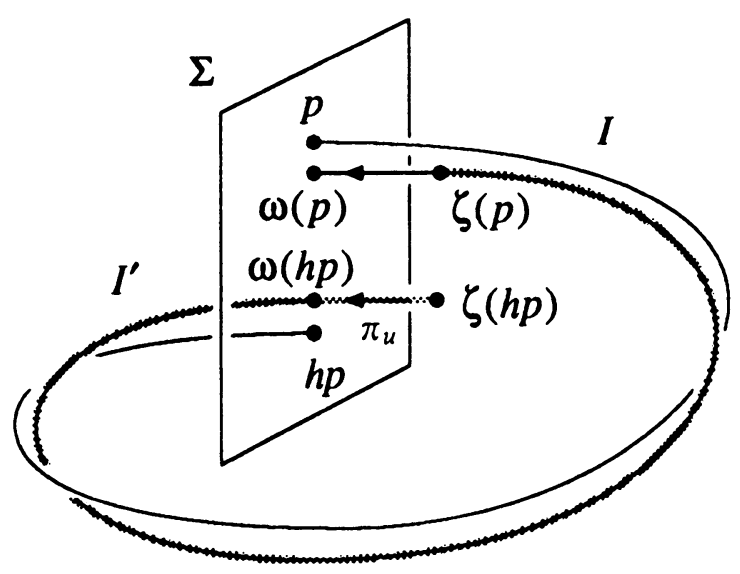

FIGURE 5

$f^{-n}(q)$ are very close. However, $g^{-n} \circ \zeta=\zeta \circ f^{-n}$, and so this is a contradiction. Therefore, $p$ and $q$ are on the same $g$ unstable manifold.

Now assume $p \in \Sigma$, and let $I$ be the segment of the $f$ strong unstable manifold connecting $p$ to $h(p)$. Define $I^{\prime}=\zeta(I)$. By the above argument, $I^{\prime}$ is a segment of a $g$ unstable manifold. This interval has end points $\zeta(p)$ and $\zeta(h(p))$ (see Figure 5). Since $p, h(p) \in \Sigma$, then $\zeta(p), \zeta(h(p)) \in U$, and $\omega(p)=\left(\pi_{u} \circ \zeta\right)(p)$ and $(\omega \circ h)(p)=\left(\pi_{u} \circ \zeta\right)(h(p))$ are well defined. $\pi_{u}$ is a projection along $g$ unstable leaves $\Rightarrow \zeta(p), \omega(p),(\zeta \circ h)(p)$, and $(\omega \circ h)(p)$ are all on the same $g$ unstable manifold. Recall $h(p)$ is defined as the first return of the $f$ strong unstable manifold through $p$ to the cross section $\Sigma$. Likewise, $\tau$ is the first return map on $\Sigma$ for the $g$ unstable foliation. Therefore, it is clear that if $\zeta$ is close enough to the identity, the first return of the $g$ unstable manifold through $\omega(p)$ to the cross section $\Sigma$ must be $(\omega \circ h)(p) \Rightarrow(\omega \circ h)(p)=(\tau \circ \omega)(p)$.

\section{Comments}

Given an appropriate initial linear Anosov on the $(k+1)$-torus (in this case, one eigenvalue greater than 1 in modulus and the remaining $k$ less than 1 and 'bunched') the above procedure would lead to a $C^{k+\alpha}$ diffeomorphism of the $k$-torus with a wandering domain [10]. In Denjoy's case $(k=1)$, it can be shown [8] that his example can be made $C^{2-\varepsilon}$ for any $\varepsilon>0$. Taken together this naturally suggests the conjecture that for any $k \in \mathbb{N}$, there exists a $C^{r}$ diffeomorphism of the $k$-torus with no periodic points and a wandering domain with dense orbit if and only if $r<k+1$.

Although there are uncountably many ergodic translations on the torus, only countably many of these can occur as semiconjugates of examples constructed as above. This follows because each example is semiconjugate to the translation induced by its original linear Anosov, and there are only countably many integer matrices. It is reasonable to assume this is an artifact of the construction since the same occurs in the case $k=1$ where it is known that a Denjoy example can be constructed from any irrational rotation; however, it is not clear how one could construct examples semiconjugate to other translations. 


\section{ACKNOWLEDGMENT}

I would like to thank Charles Pugh for his helpful comments and suggestions in the preparation of this manuscript.

\section{REFERENCES}

1. V. Akis, A Denjoy diffeomorphism of the torus, preprint.

2. P. Bohl, Über die hinsichtlich der unabhängigen und abhängigen variabeln periodische defferentialgleichung erster ordnung, Acta Math. 40 (1916), 321-336.

3. A. Denjoy, Sur les courbes définies par les équations différentielles à la surface du tore, J. Math. Pure Appl. 11 (1932), 333-375.

4. J. Harrison, Unsmoothable diffeomorphisms, Ann. of Math. (2) 102 (1975), 83-94.

5. __ Unsmoothable diffeomorphisms on higher dimensional manifolds, Proc. Amer. Math. Soc. 73 (1979), 249-255.

6. _ـ Denjoy fractals, Topology 28 (1989), 69-80.

7. $\ldots$ Embedded continued fractals and their Hausdorff dimension, Constr. Approx. 5 (1989), 99-115.

8. M. Herman, Sur la conjugaison différentiable des difféomorphismes du cercle à des rotations, Inst. Hautes Études Sci. Publ. Math. 49 (1979), 1-233.

9. M. Hirsch, C. Pugh, and M. Shub, Invariant manifolds, Lecture Notes in Math., vol. 583, Springer-Verlag, New York, 1977.

10. P. McSwiggen, Diffeomorphisms of the $k$-torus with wandering domains, preprint.

11. A. Norton and J. Velling, Denjoy-like theorems for diffeomorphisms of the 2-torus, preprint.

12. P. Walters, Anosov diffeomorphisms are topologically stable, Topology 9 (1970), 71-78.

Department of Mathematical Sciences, The University of Cincinnati, Cincinnati, ОHIо 45221-0025

E-mail address: pat.mcswiggen@uc.edu 